\title{
Vasoactive Thiomethyl-Pyrimidines: Promising Drug Candidates with Vascular Activity
}

\author{
Audrey N. de Andrade, ${ }^{a}$ Alice V. Araújo, ${ }^{b}$ Hugo B. W. Barbosa, ${ }^{a}$ Almir G. Wanderley, ${ }^{c}$ \\ Oscar L. Malta*,a and Janaína V. dos Anjos $*, a$
}

\author{
${ }^{a}$ Departamento de Química Fundamental, Universidade Federal de Pernambuco, \\ 50740-560 Recife-PE, Brazil
}

${ }^{b}$ Núcleo de Nutrição, Universidade Federal de Pernambuco, 55608-680 Vitória de Santo Antão-PE, Brazil

\author{
'Departamento de Fisiologia e Farmacologia, Universidade Federal de Pernambuco, \\ 50670-901 Recife-PE, Brazil
}

\begin{abstract}
Pyrimidines and their derivatives are present in various biologically active molecules. Most of the synthetic methods employed to achieve the pyrimidinone ring consist of two stages: the synthesis of a Michael intermediate from an aldehyde and an "active methylene" containing compound; and the condensation of this intermediate with a molecule containing an uranium moiety. This may take one to two days of laboratory work. In this paper we describe a new methodology in which these derivatives are obtained via multicomponent synthesis mediated by ultrasound in only 2 hours. In order to obtain water-soluble pyrimidinone derivatives, our previous compounds were further converted into their sodium salts. In pharmacologic studies, these salts inhibited phenylephrineinduced contraction in isolated rat aorta, suggesting that they may act as alpha- 1 antagonists and, therefore, are candidates for anti-hypertensive drugs.
\end{abstract}

Keywords: pyrimidines, vascular activity, hypertension, drug discovery, ultrasound

\section{Introduction}

Alpha adrenergic receptors are involved in many biological actions of catecholamines. Therefore, those receptors have been explored as targets for a large number of substances and they have been employed for the treatment of several pathologies. The most important effects of alpha adrenergic antagonists are related to the cardiovascular system. ${ }^{1,2}$

The catecholamines receptors can be classified according to their binding specificities, formerly alpha-1 and alpha-2. ${ }^{1}$ They are quite similar and belong to $\mathrm{G}$ protein-coupled superfamily receptors. ${ }^{1,3}$ While alpha-1 adrenergic receptors mediate catecholamine-induced peripheral vasoconstriction, alpha- 2 is mainly involved in the peripheral and central regulation of the sympathetic nervous system. Alpha-1 receptors can also be divided into three subtypes, alpha 1A, alpha 1B, and alpha 1D, that have distinct actions and tissue expression. ${ }^{3,4}$

*e-mail: omlmalta@gmail.com; janaina.anjos@ufpe.br
When an alpha- 1 adrenergic receptor is blocked by an antagonist, it inhibits the contraction of blood vessels and vasodilation takes place. This results in blood pressure drop. For this reason, alpha-1 adrenergic receptor antagonists are mainly used as anti-hypertensive drugs. ${ }^{5}$ These drugs include Prazosin (I), Terazosin (II) and Doxazosin (III) (Figure 1), that are quite similar in their structures, belonging to the 2,4-diamino-6,7-dimethoxyquinazolines class of AR-1 receptor antagonists. ${ }^{1,4}$

It can be observed that Prazosin, Terazosin and Doxazosin have a pyrimidine ring in their chemical structures. ${ }^{4}$ In fact, pyrimidines and their oxo-derivatives are described in the literature as presenting a wide array of biological activities such as antitumor, ${ }^{6,7}$ interferon inducer, ${ }^{8}$ antiviral, ${ }^{9}$ anti-hipertensive, ${ }^{10}$ hypoglycemic, ${ }^{11}$ anticonvulsant, ${ }^{12}$ antinociceptive, ${ }^{13,14}$ and anti-inflammatory. ${ }^{15}$

Pyrimidine or pyrimidinone rings can be synthesized in several ways. ${ }^{16}$ Most often, for the synthesis of these type of heterocycles, a Michael adduct and a uronium-containing molecule (guanidine, ${ }^{17}$ amidines, ${ }^{18}$ urea, ${ }^{19}$ thiourea and their derivative ${ }^{20}$ ) react in the presence of an organic base. 
<smiles>COc1cc2nc(N3CCN(C(=O)c4ccco4)CC3)nc(N)c2cc1OC</smiles><smiles>COc1cc2nc(N3CCN(C(=O)C4CCCO4)CC3)nc(N)c2cc1OC</smiles><smiles>COc1cc2nc(N3CCN(C(=O)C4COc5ccccc5O4)CC3)nc(N)c2cc1OC</smiles>

Figure 1. Molecular structures of Prazosin (I), Terazosin (II) and Doxazosin (III).

This reaction results in an alicyclic compound that can be converted into the heterocycles of interest employing cyclization conditions. This whole process may take two days of laboratory work for the preparation of one product. ${ }^{9-11,14,18}$ These findings encouraged us to search new ways to perform these reactions and to evaluate the biological activity of these heterocyclic derivatives.

Multicomponent reactions (MCRs) are procedures in which three or more reagents are mixed together to yield a product, in a domino process. ${ }^{21}$ These types of reaction can be efficiently accomplished by employing ultrasonic irradiation. ${ }^{22,23}$ The use of ultrasound energy in organic reactions relies on the cavitation effect, which induces mass transfer enhancement, leading to better reaction yields and shorter reaction times. Using mild conditions and sonochemistry, a lot of heterocyclic rings have been synthesized, including pyrimidines and their derivatives. ${ }^{24-27}$ As an example, Pagadala et al. ${ }^{28}$ have used ultrasound irradiation to synthesize pyrimidines in aqueous $\mathrm{NaOH}$ solution, by addition of aromatic aldehyde to acetyl acetone and thiourea, via multicomponent approach.

These positive impacts on MCRs by the use of ultrasound irradiation have prompted us to investigate and to compare the methodologies (sonochemistry and conventional stirring) in the synthesis of thiomethylpyrimidinones. This study also aimed to evaluate whether the synthesized compounds have alpha-1 antagonist action in vascular reactivity studies.

\section{Experimental}

\section{General information}

All commercially available reagents were used without any further purification and the reactions were monitored by thin layer chromatography (TLC) analysis with TLC plates containing $\mathrm{GF}_{254}$ (E. Merck). The reactions were carried out in a Branson B2510E-DTH ultrasonic bath operating at frequency of $40 \mathrm{kHz}$. Melting points were determined on a Büchi apparatus and are uncorrected. Nuclear magnetic resonance (NMR) spectra were recorded with a Varian UNMRS $400 \mathrm{MHz}$ spectrometer. Infrared spectra were obtained on a Brucker IFS66 FT-IR, using $\mathrm{KBr}$ pellets. Exact mass measurements of the molecular ions were obtained on a Shimadzu Electrospray LC/MS-IT-TOF. An elemental analysis was performed with a Carlo Erba instrument model E-1110.

Multicomponent synthesis of 2-methylsulfanyl-6-oxo-4-aryl1,6-dihydro-pyrimidine 5-carbonitriles (4a-j)

To an Erlenmeyer (capacity: $50 \mathrm{~mL}$ ), were added $1.2 \mathrm{mmol}(108 \mathrm{mg})$ of 2-methyl-2-thiopseudourea hemisulfate and $2 \mathrm{mmol}(276 \mathrm{mg}$ ) of potassium carbonate dissolved in $4 \mathrm{~mL}$ of distilled water. This mixture was sonicated at room temperature until the neutralization of the 2-methyl-2-thiopseudourea salt. To a beaker (capacity: $20 \mathrm{~mL}$ ), $1 \mathrm{mmol}$ of the corresponding aromatic aldehyde and $1.2 \mathrm{mmol}(136 \mathrm{mg}, 0.13 \mathrm{~mL}$ ) of ethyl cyanoacetate were dissolved in $4 \mathrm{~mL}$ of ethanol. This solution was added to the Erlenmeyer containing the 2-methyl-2-thiopseudourea basic solution. The reaction mixture was sonicated at a frequency of $40 \mathrm{kHz}$ at $50{ }^{\circ} \mathrm{C}$ for 2 hours. The product was precipitated by addition of concentrated $\mathrm{HCl}$ and poured into ice. The formed precipitate was then vacuum filtered, washed with distilled water $(50 \mathrm{~mL})$, and recrystallized from ethanol to provide the pure pyrimidinones.

2-Methylsulfanyl-6-oxo-4-phenyl-1,6-dihydro-pyrimidine5-carbonitrile (4a)

White crystals; yield $72 \%$; m.p. $280-282{ }^{\circ} \mathrm{C}(\mathrm{EtOH})$; $\mathrm{R}_{\mathrm{f}} 0.84$ (9:1 $\left.\mathrm{CHCl}_{3} / \mathrm{MeOH} \mathrm{v} / \mathrm{v}\right)$; IR (KBr pellet) $v_{\max } / \mathrm{cm}^{-1}$ 
$2854\left(\mathrm{CH}_{\text {arom }}\right), 2220(\mathrm{C} \equiv \mathrm{N}), 1662(\mathrm{C}=\mathrm{O}), 1540(\mathrm{C}=\mathrm{N})$; ${ }^{1} \mathrm{H}$ NMR $\left(400 \mathrm{MHz}, \mathrm{DMSO}-d_{6}\right) \delta 2.61\left(\mathrm{~s}, 3 \mathrm{H}, \mathrm{SCH}_{3}\right), 7.54-$ 7.62 (m, 3H, Ph-H), 7.95 (dd, 1H, J 2.4, $8.1 \mathrm{~Hz}, \mathrm{Ph}-\mathrm{H}$ ), 7.97 (dd, $1 \mathrm{H}, J 2.4,8.1 \mathrm{~Hz}, \mathrm{Ph}-\mathrm{H}) ;{ }^{13} \mathrm{C}$ NMR $(100 \mathrm{MHz}$, DMSO- $\left.d_{6}\right) \delta 13.8,93.4,116.4,129.1,126.2,132.2$, 135.8, 161.4, 167.1, 167.7; HRMS-ESI (high resolution mass spectrometry-electrospray ionization) $\mathrm{m} / \mathrm{z}$ calcd. for $\mathrm{C}_{12} \mathrm{H}_{8} \mathrm{~N}_{3} \mathrm{OS}[\mathrm{M}-\mathrm{H}]^{+}: 242.0388$, found: 242.0081 .

2-Methylsulfanyl-6-oxo-4-o-tolyl-1,6-dihydro-pyrimidine5-carbonitrile (4b)

White crystals; yield 55\%; m.p. $205-207{ }^{\circ} \mathrm{C}(\mathrm{EtOH})$; $\mathrm{R}_{\mathrm{f}} 0.68$ (9:1 $\mathrm{CHCl}_{3} / \mathrm{MeOH}$ v/v); IR (KBr) $v_{\max } / \mathrm{cm}^{-1} 2843$, 2224, 1665, 1546; ${ }^{1} \mathrm{H}$ NMR (400 MHz, DMSO- $\left.d_{6}\right) \delta 2.31$ (s, $\left.3 \mathrm{H}, \mathrm{PhCH}_{3}\right), 2.52$ (s, 3H, $\left.\mathrm{SCH}_{3}\right), 7.30-7.45(\mathrm{~m}, 4 \mathrm{H}$, $\mathrm{Ph}-\mathrm{H}) ;{ }^{13} \mathrm{C}$ NMR (100 MHz, DMSO- $\left.d_{6}\right) \delta 13.0,19.1,95.8$, 114.9, 125.6, 128.4, 130.0, 130.5, 135.4, 166.6; HRMS-ESI $m / z$ calcd. for $\mathrm{C}_{13} \mathrm{H}_{10} \mathrm{~N}_{3} \mathrm{O}_{2} \mathrm{~S}[\mathrm{M}-\mathrm{H}]^{+}: 256.0545$, found: 256.0243 .

2-Methylsulfanyl-6-oxo-4-p-tolyl-1,6-dihydro-pyrimidine5-carbonitrile (4c)

White crystals; yield 50\%; m.p. $285-288{ }^{\circ} \mathrm{C}(\mathrm{EtOH})$; $\mathrm{R}_{\mathrm{f}} 0.79$ (9:1 $\left.\mathrm{CHCl}_{3} / \mathrm{MeOH} \mathrm{v} / \mathrm{v}\right)$; IR (KBr) $v_{\max } / \mathrm{cm}^{-1} 2754$, 2221, 1652, 1523; ${ }^{1} \mathrm{H}$ NMR (400 MHz, DMSO- $\left.d_{6}\right) \delta 2.39$ (s, $\left.3 \mathrm{H}, \mathrm{PhCH}_{3}\right), 2.60\left(\mathrm{~s}, 3 \mathrm{H}, \mathrm{SCH}_{3}\right), 7.37(\mathrm{~d}, 2 \mathrm{H}, J 10.8 \mathrm{~Hz}$, $\mathrm{Ph}-\mathrm{H}), 7.89$ (d, 2H, $J 10.8 \mathrm{~Hz}, \mathrm{Ph}-\mathrm{H}) ;{ }^{13} \mathrm{C}$ NMR $(100 \mathrm{MHz}$, DMSO- $\left.d_{6}\right) \delta 13.7,21.6,92.8,116.6,129.2,129.6,132.9$, 142.6, 161.4, 166.8, 167.4; HRMS-ESI $\mathrm{m} / \mathrm{z}$ calcd. for $\mathrm{C}_{13} \mathrm{H}_{10} \mathrm{~N}_{3} \mathrm{O}_{2} \mathrm{~S}[\mathrm{M}-\mathrm{H}]^{+}:$256.0545, found: 256.0245 .

2-Methylsulfanyl-6-oxo-4-p-chlorophenyl-1,6-dihydropyrimidine-5-carbonitrile (4d)

White crystals; yield 47\%; m.p. 293-295 ${ }^{\circ} \mathrm{C}(\mathrm{EtOH})$; $\mathrm{R}_{\mathrm{f}} 0.54\left(9: 1 \mathrm{CHCl}_{3} / \mathrm{MeOH} \mathrm{v} / \mathrm{v}\right)$; IR $(\mathrm{KBr}) v_{\max } / \mathrm{cm}^{-1}$ 2865, 2222, 1671, 1546; ${ }^{1} \mathrm{H}$ NMR (400 MHz, DMSO- $d_{6}$ ) $\delta 2.60\left(\mathrm{~s}, 3 \mathrm{H}, \mathrm{SCH}_{3}\right), 3.43(\mathrm{~s}, 1 \mathrm{H}, \mathrm{NH}), 7.64(\mathrm{dd}, 2 \mathrm{H}$, $J 8.4 \mathrm{~Hz}, \mathrm{Ph}-\mathrm{H}$ ), 7.98 (dd, 2H, J $8.4 \mathrm{~Hz}, \mathrm{Ph}-\mathrm{H}) ;{ }^{13} \mathrm{C} \mathrm{NMR}$ $\left(100 \mathrm{MHz}, \mathrm{DMSO}-d_{6}\right) \delta 13.3,93.0,115.8,128.7$, 130.6, 134.1, 136.6, 161.0, 165.9, 167.0; HRMS-ESI $m / z$ calcd. for $\mathrm{C}_{12} \mathrm{H}_{8} \mathrm{ClN}_{3} \mathrm{NaOS}[\mathrm{M}+\mathrm{Na}]^{+}:$299.9974, found: 299.9821.

2-Methylsulfanyl-6-oxo-4- $p$-bromophenyl-1,6-dihydropyrimidine-5-carbonitrile (4e)

White crystals; yield $40 \%$; m.p. $>300{ }^{\circ} \mathrm{C}(\mathrm{EtOH})$; $\mathrm{R}_{\mathrm{f}} 0.75\left(9: 1 \mathrm{CHCl}_{3} / \mathrm{MeOH} \mathrm{v} / \mathrm{v}\right)$; IR $(\mathrm{KBr}) v_{\max } / \mathrm{cm}^{-1}$ 2927, 2215, 1650, 1572; ${ }^{1} \mathrm{H}$ NMR (400 MHz, DMSO- $d_{6}$ ) $\delta 2.61$ (s, 3H, $\left.\mathrm{SCH}_{3}\right), 7.79$ (d, 2H, J 8.4 Hz, Ph-H), 7.91 (d, 2H, J $8.4 \mathrm{~Hz}, \mathrm{Ph}-\mathrm{H}), 13.77$ (s, 1H, NH); ${ }^{13} \mathrm{C} \mathrm{NMR}$ (100 MHz, DMSO- $\left.d_{6}\right) \delta 13.3,93.0,115.7,125.5,130.6$,
131.6, 134.4, 160.8, 166.0, 166.8; HRMS-ESI $\mathrm{m} / \mathrm{z}$ calcd. for $\mathrm{C}_{12} \mathrm{H}_{8} \mathrm{BrKN}_{3} \mathrm{OS}[\mathrm{M}+\mathrm{K}]^{+}: 361.2787$, found: 361.8388 .

2-Methylsulfanyl-6-oxo-4-p-fluorophenyl-1,6-dihydropyrimidine-5-carbonitrile (4f)

White crystals; yield 56\%; m.p. $283-285^{\circ} \mathrm{C}(\mathrm{EtOH})$; $\mathrm{R}_{\mathrm{f}} 0.58\left(9: 1 \mathrm{CHCl}_{3} / \mathrm{MeOH} \mathrm{v} / \mathrm{v}\right)$; IR ( $\left.\mathrm{KBr}\right) \mathrm{v}_{\max } / \mathrm{cm}^{-1}$ 2835, 2223, 1655, 1536; ${ }^{1} \mathrm{H}$ NMR (400 MHz, DMSO- $d_{6}$ ) $\delta 2.59$ (s, 3H, $\left.\mathrm{SCH}_{3}\right), 7.39$ (d, 2H, J $\left.8.4 \mathrm{~Hz}, \mathrm{Ph}-\mathrm{H}\right), 8.10$ (d, $2 \mathrm{H}, J 8.4 \mathrm{~Hz}, \mathrm{Ph}-\mathrm{H}), 13.76$ (s, 1H, NH); ${ }^{13} \mathrm{C}$ NMR $\left(100 \mathrm{MHz}, \mathrm{DMSO}-d_{6}\right) \delta 13.3,92.8,115.8,115.9,131.4$, $131.5,131.7,160.8,165.3,165.3,165.7,166.6$; HRMSESI $m / z$ calcd. for $\mathrm{C}_{12} \mathrm{H}_{8} \mathrm{FN}_{3} \mathrm{NaOS}\left[\mathrm{M}+\mathrm{Na}^{+}: 284.0270\right.$, found: 283.9578 .

4-(4-Methoxy-phenyl)-2-methylsulfanyl-6-oxo-1,6-dihydropyrimidine-5-carbonitrile $\mathbf{( 4 g )}$

White crystals; yield 55\%; m.p. $280-283{ }^{\circ} \mathrm{C}(\mathrm{EtOH})$; $\mathrm{R}_{\mathrm{f}} 0.78$ (9:1 $\left.\mathrm{CHCl}_{3} / \mathrm{MeOH} \mathrm{v} / \mathrm{v}\right)$; IR (KBr) $v_{\max } / \mathrm{cm}^{-1} 2841$, $2216,1650,1606 ;{ }^{1} \mathrm{H}$ NMR $\left(400 \mathrm{MHz}\right.$, DMSO- $\left.d_{6}\right) \delta 2.60$ (s, $3 \mathrm{H}, \mathrm{SCH}_{3}$ ), 3.85 (s, $\left.3 \mathrm{H}, \mathrm{PhOCH}_{3}\right), 7.10$ (dd, $2 \mathrm{H}, J$ 2.5, $9.3 \mathrm{~Hz}, \mathrm{Ph}-\mathrm{H}), 7.11$ (dd, 2H, J 2.5, $9.3 \mathrm{~Hz}, \mathrm{Ph}-\mathrm{H}), 8.02$ (dd, 2H, J 2.5, 9.3 Hz, Ph-H), 8.02 (dd, 2H, J 2.5, $9.3 \mathrm{~Hz}$, $\mathrm{Ph}-\mathrm{H}) ;{ }^{13} \mathrm{C}$ NMR $\left(100 \mathrm{MHz}, \mathrm{DMSO}-d_{6}\right) \delta 13.7,56.0,91.7$, 114.5, 116.9, 127.7, 131.3, 162.7, 166.4, 166.6; HRMS-ESI $\mathrm{m} / z$ calcd. for $\mathrm{C}_{13} \mathrm{H}_{12} \mathrm{~N}_{3} \mathrm{O}_{2} \mathrm{~S}[\mathrm{M}+\mathrm{H}]^{+}: 274.0650$, found: 274.0305 .

2-Methylsulfanyl-6-oxo-4-m-nitrophenyl-1,6-dihydropyrimidine-5-carbonitrile (4h)

White crystals; yield 53\%; m.p. 258-263 ${ }^{\circ} \mathrm{C}(\mathrm{EtOH})$; $\mathrm{R}_{\mathrm{f}} 0.41\left(9: 1 \mathrm{CHCl}_{3} / \mathrm{MeOH} \mathrm{v} / \mathrm{v}\right)$; IR (KBr) $v_{\max } / \mathrm{cm}^{-1} 2860$, $2225,1669,1543 ;{ }^{1} \mathrm{H}$ NMR (400 MHz, DMSO- $\left.d_{6}\right) \delta 2.63$ (s, $\left.3 \mathrm{H}, \mathrm{SCH}_{3}\right), 7.88$ (dd, $\left.1 \mathrm{H}, J 7.6 \mathrm{~Hz}, \mathrm{Ph}-\mathrm{H}\right), 8.41$ (dd, $2 \mathrm{H}, J$ 7.6, 7.6 Hz, Ph-H), 8.74 (s, $1 \mathrm{H}, \mathrm{Ph}-\mathrm{H}) ;{ }^{13} \mathrm{C}$ NMR $\left(100 \mathrm{MHz}, \mathrm{DMSO}-d_{6}\right) \delta 13.2,93.8,115.3,123.1,125.9$, 130.3, 134.8, 136.7, 147.7, 160.8, 164.8, 167.5; HRMS-ESI $\mathrm{m} / z$ calcd. for $\mathrm{C}_{12} \mathrm{H}_{8} \mathrm{KN}_{4} \mathrm{O}_{3} \mathrm{~S}[\mathrm{M}+\mathrm{K}]^{+}: 326.9954$, found: 326.9211 .

2-Methylsulfanyl-6-oxo-4-p-nitrophenyl-1,6-dihydropyrimidine-5-carbonitrile (4i)

Yellow crystals; yield 53\%; m.p. 290-293 ${ }^{\circ} \mathrm{C}(\mathrm{EtOH})$; $\mathrm{R}_{\mathrm{f}} 0.33\left(9: 1 \mathrm{CHCl}_{3} / \mathrm{MeOH} \mathrm{v} / \mathrm{v}\right)$; IR (KBr) $v_{\max } / \mathrm{cm}^{-1} 3101$, 2230, 1696, 1525; ${ }^{1} \mathrm{H}$ NMR (400 MHz, DMSO- $\left.d_{6}\right) \delta 2.60$ (s, $3 \mathrm{H}, \mathrm{SCH}_{3}$ ), 3.35 (s, $\left.3 \mathrm{H}, \mathrm{PhOCH}_{3}\right), 8.20$ (dd, $2 \mathrm{H}, J$ 2.5, $8.8 \mathrm{~Hz}, \mathrm{Ph}-\mathrm{H}), 8.40$ (dd, $2 \mathrm{H}, J 2.5,8.8 \mathrm{~Hz}, \mathrm{Ph}-\mathrm{H}) ;{ }^{13} \mathrm{C} \mathrm{NMR}$ $\left(100 \mathrm{MHz}, \mathrm{DMSO}-d_{6}\right) \delta 13.4,115.5,123.2,129.4,130.2$, 141.3, 149.0, 165.4, 167.6. Anal. calcd. for $\mathrm{C}_{12} \mathrm{H}_{8} \mathrm{~N}_{4} \mathrm{O}_{3} \mathrm{~S}$ : C, 50.00; H, 2.80; N, 19.43; S, 11.12\%; found: C, 49.64; H, 3.08; N, 19.45; S, $10.71 \%$. 
4-(3,4-Dichloro-phenyl)-2-methylsulfanyl-6-oxo-1,6-dihydropyrimidine-5-carbonitrile $(4 \mathbf{j})$

White crystals; yield $40 \%$; m.p. $297-300{ }^{\circ} \mathrm{C}(\mathrm{EtOH})$; $\mathrm{R}_{\mathrm{f}} 0.53$ (9:1 $\left.\mathrm{CHCl}_{3} / \mathrm{MeOH} \mathrm{v} / \mathrm{v}\right)$; IR (KBr) $v_{\max } / \mathrm{cm}^{-1} 2856$, 2224, 1666, 1549; ${ }^{1} \mathrm{H}$ NMR (400 MHz, DMSO- $\left.d_{6}\right) \delta 2.61$ $\left(\mathrm{s}, 3 \mathrm{H}, \mathrm{SCH}_{3}\right), 3.35(\mathrm{~s}, 1 \mathrm{H}, \mathrm{NH}), 7.84(\mathrm{~d}, 1 \mathrm{H}, J 8.0 \mathrm{~Hz}$, $\mathrm{Ph}-\mathrm{H}), 7.93$ (d, 1H, J 8.0 Hz, Ph-H), 8.15 (s, 1H, $\mathrm{H}_{\text {arom }}$ ); ${ }^{13} \mathrm{C}$ NMR $\left(400 \mathrm{MHz}\right.$, DMSO- $\left.d_{6}\right) \delta 13.2,93.5,115.3,128.6$, 130.3, 130.8, 131.4, 134.4, 135.6, 160.6, 164.4, 167.2. Anal. calcd. for $\mathrm{C}_{12} \mathrm{H}_{7} \mathrm{C}_{12} \mathrm{~N}_{3} \mathrm{OS}$ : C, 46.17; H, 2.26; N, 13.46; S, $10.27 \%$; found: C, $46.02 ; \mathrm{H}, 2.54 ; \mathrm{N}, 13.38 ; \mathrm{S}, 9.45 \%$.

Synthesis of 2-methylsulfanyl-6-oxo-4-aryl-1,6-dihydropyrimidine 5 -carbonitriles sodium salts $(\mathbf{5 a}-\mathbf{j})$

To an Erlenmeyer (capacity: $50 \mathrm{~mL}$ ) containing $1 \mathrm{mmol}$ of the corresponding pyrimidinone derivative (4a-j), were added $1 \mathrm{mmol}$ of $\mathrm{NaOH}$ dissolved in $20 \mathrm{~mL}$ of ethanol. The reaction mixture was sonicated at a frequency of $40 \mathrm{kHz}$ for 30 minutes. The contents were then evaporated and the formed precipitate was recrystallized from a methanol/diethyl ether mixture to afford the corresponding sodium salts.

2-Methylsulfanyl-6-oxo-4-phenyl-1,6-dihydro-pyrimidine5 -carbonitrile sodium salt ( $5 \mathbf{a})$

White crystals; IR (KBr) $v_{\max } / \mathrm{cm}^{-1} 2204,1541,1490$; ${ }^{1} \mathrm{H}$ NMR (400 MHz, $\left.\mathrm{D}_{2} \mathrm{O}\right) \delta 2.51$ (s, 3H, $\left.\mathrm{SCH}_{3}\right), 7.51-7.59$ (m, 3H, Ph-H), 7.73 (dd, 2H, J 2.8, 8.4 Hz, Ph-H); ${ }^{13} \mathrm{C} \mathrm{NMR}$ $\left(100 \mathrm{MHz}, \mathrm{DMSO}-d_{6}\right) \delta 13.3,88.6,120.1,128.1,128.1$, 129.7, 137.7, 166.9, 170.6, 172.4.

2-Methylsulfanyl-6-oxo-4-o-tolyl-1,6-dihydro-pyrimidine5 -carbonitrile sodium salt (5b)

White crystals; IR (KBr pellet) $v_{\max } / \mathrm{cm}^{-1} 2221,1649$, 1528; 'H NMR (400 MHz, DMSO- $\left.d_{6}\right) \delta 2.30$ (s, 3H, $\mathrm{PhCH}_{3}$ ), 2.51 (s, 3H, $\left.\mathrm{SCH}_{3}\right), 7.30-7.46$ (m, 4H, Ph-H); ${ }^{13} \mathrm{C}$ NMR $\left(100 \mathrm{MHz}, \mathrm{DMSO}-d_{6}\right) \delta 13.2,19.3,95.9,115.1,125.7$, 128.6, 130.2, 130.7, 135.5, 135.7, 160.4, 166.6, 194.4.

2-Methylsulfanyl-6-oxo-4-p-tolyl-1,6-dihydro-pyrimidine5 -carbonitrile sodium salt $(\mathbf{5 c})$

White crystals; IR (KBr) $v_{\max } / \mathrm{cm}^{-1} 2923,2210$, 1650, 1543; ${ }^{1} \mathrm{H}$ NMR (400 MHz, DMSO- $d_{6}$ ) $\delta 2.38$ (s, $\left.3 \mathrm{H}, \mathrm{PhCH}_{3}\right), 2.06$ (s, $\left.3 \mathrm{H}, \mathrm{SCH}_{3}\right), 7.35(\mathrm{~d}, 2 \mathrm{H}, J 8.0 \mathrm{~Hz}$, $\mathrm{Ph}-\mathrm{H}), 7.88$ (d, 2H, J 8.0 Hz, Ph-H); ${ }^{13} \mathrm{C}$ NMR $(100 \mathrm{MHz}$, DMSO- $\left.d_{6}\right) \delta 13.2,21.0,92.3,116.0,128.7,128.8,129.1$, 132.4, 142.1, 160.9, 166.3, 166.8 .

2-Methylsulfanyl-6-oxo-4-p-chlorophenyl-1,6-dihydropyrimidine-5-carbonitrile sodium salt $\mathbf{( 5 d}$ )

White crystals; IR (KBr) $v_{\max } / \mathrm{cm}^{-1} 2200,1663,1547$;
${ }^{1} \mathrm{H}$ NMR (400 MHz, DMSO- $\left.d_{6}\right) \delta 2.35$ (s, 3H, $\mathrm{SCH}_{3}$ ), 7.52 (d, 2H, J $8.8 \mathrm{~Hz}, \mathrm{Ph}-\mathrm{H}), 7.79$ (d, 2H, J $8.8 \mathrm{~Hz}, \mathrm{Ph}-\mathrm{H}$ ); ${ }^{13} \mathrm{C}$ NMR $\left(100 \mathrm{MHz}\right.$, DMSO- $\left.d_{6}\right) \delta 13.3,88.4,119.9$, $128.17,129.9,134.4,136.5,165.6,170.5,172.64$.

2-Methylsulfanyl-6-oxo-4-p-bromophenyl-1,6-dihydropyrimidine-5-carbonitrile sodium salt (5e)

White crystals; IR (KBr) $v_{\max } / \mathrm{cm}^{-1} 3434,2208,1546$, 1481; ${ }^{1} \mathrm{H}$ NMR (300 MHz, $\left.\mathrm{D}_{2} \mathrm{O}\right) \delta 2.51\left(\mathrm{~s}, 3 \mathrm{H}, \mathrm{SCH}_{3}\right.$ ), 7.65 (d, 2H, J 9.0 Hz, Ph-H), 7.71 (d, 2H, J 9.0 Hz, Ph-H); ${ }^{13} \mathrm{C}$ NMR $\left(100 \mathrm{MHz}\right.$, DMSO- $\left.d_{6}\right) \delta 13.3,88.4,119.9,123.2$, 130.2, 131.1, 136.8, 165.7, 170.58, 172.7.

2-Methylsulfanyl-6-oxo-4- $p$-fluorophenyl-1,6-dihydropyrimidine-5-carbonitrile sodium salt (5f)

White crystals; IR (KBr) $v_{\max } / \mathrm{cm}^{-1} 3068,2204,1550$, $1483(\mathrm{C}=\mathrm{N}) ;{ }^{1} \mathrm{H}$ NMR $\left(400 \mathrm{MHz}, \mathrm{D}_{2} \mathrm{O}\right) \delta 2.52(\mathrm{~s}, 3 \mathrm{H}$, $\left.\mathrm{SCH}_{3}\right)$, 7.24-7.31 (m, 2H, Ph-H), 7.77-7.82 (m, 2H, Ph-H); ${ }^{13} \mathrm{C}$ NMR $\left(100 \mathrm{MHz}\right.$, DMSO- $\left.d_{6}\right) \delta 13.3,88.3,114.9,115.1$, 120.2, 130.4, 130.5, 134.2, 134.16, 161.6, 164.1, 165.7, $170.5,172.5$.

4-(4-Methoxy-phenyl)-2-methylsulfanyl-6-oxo-1,6-dihydropyrimidine-5-carbonitrile sodium salt $\mathbf{( 5 g}$ )

White crystals; IR (KBr pellet) $v_{\text {max }} / \mathrm{cm}^{-1} 2217,1649$, 1606; ' ${ }^{\mathrm{H}} \mathrm{NMR}\left(400 \mathrm{MHz}, \mathrm{DMSO}-d_{6}\right) \delta 2.60\left(\mathrm{~s}, 3 \mathrm{H}, \mathrm{SCH}_{3}\right.$ ), 3.85 (s, 3H, $\mathrm{PhOCH}_{3}$ ), 7.11 (dd, $2 \mathrm{H}, J 8.8 \mathrm{~Hz}, \mathrm{Ph}-\mathrm{H}$ ), 8.0 (dd, $2 \mathrm{H}, J 8.8 \mathrm{~Hz}, \mathrm{Ph}-\mathrm{H}) ;{ }^{13} \mathrm{C}$ NMR (100 MHz, DMSO- $d_{6}$ ) $\delta 13.4,88.6,119.8,128.3,129.9,130.5,130.9,132.4$, 138.2, 164.1, 170.1, 172.7.

2-Methylsulfanyl-6-oxo-4-m-nitrophenyl-1,6-dihydropyrimidine-5-carbonitrile sodium salt $(\mathbf{5 h})$

White crystals; IR (KBr) $v_{\max } / \mathrm{cm}^{-1} 2202,1528,1548$; ${ }^{1} \mathrm{H} \mathrm{NMR}\left(400 \mathrm{MHz}\right.$, DMSO- $\left.d_{6}\right) \delta 2.20\left(\mathrm{~s}, 3 \mathrm{H}, \mathrm{SCH}_{3}\right), 7.59$ (dd, 2H, J 8.0, 8.0 Hz, Ph-H), 8.10 (d, 2H, J 8.4 Hz, Ph-H), 8.15 (d, 2H, J 8.4 Hz, Ph-H), 8.44 (s, 1H, Ph-H); ${ }^{13} \mathrm{C}$ NMR $\left(100 \mathrm{MHz}, \mathrm{DMSO}-d_{6}\right) \delta$ 13.3, 115.6, 123.3, 126.0 130.4, 134.9, 136.8, 147.7, 164.9, 167.6.

2-Methylsulfanyl-6-oxo-4-p-nitrophenyl-1,6-dihydropyrimidine-5-carbonitrile sodium salt (5i)

Yellow crystals; IR (KBr) $v_{\max } / \mathrm{cm}^{-1} 2223,1675,1527$; ${ }^{1} \mathrm{H}$ NMR $\left(300 \mathrm{MHz}, \mathrm{D}_{2} \mathrm{O}\right) \delta 2.51$ (s, 3H, $\mathrm{SCH}_{3}$ ), 7.95 (d, $2 \mathrm{H}, J 8.4 \mathrm{~Hz}, \mathrm{Ph}-\mathrm{H}), 8.37$ (d, 2H, J 8.4 Hz, Ph-H); ${ }^{13} \mathrm{C}$ NMR $\left(100 \mathrm{MHz}, \mathrm{DMSO}-d_{6}\right) \delta 13.3,89.4,119.3,123.4,129.6$, 143.7, 148.0, 165.0, 169.7, 172.7.

4-(3,4-Dichloro-phenyl)-2-methylsulfanyl-6-oxo-1,6-dihydropyrimidine-5-carbonitrile sodium salt (5j)

White crystals; IR (KBr) $v_{\max } / \mathrm{cm}^{-1} 2202,1528,1488$; 
${ }^{1} \mathrm{H}$ NMR (400 MHz, DMSO- $d_{6}$ ) $\delta 2.61$ (s, 3H, $\mathrm{SCH}_{3}$ ), 7.92 (dd, 1H, J 2.0, 8.0 Hz, Ph-H), 7.94 (dd, $1 \mathrm{H}, J 2.0,8.0 \mathrm{~Hz}$, $\mathrm{Ph}-\mathrm{H}), 8.15$ (d, 1H, J 8.0 Hz, Ph-H); ${ }^{13} \mathrm{C}$ NMR (100 MHz, DMSO- $\left.d_{6}\right) \delta 13.3,93.65,115.6,128.8,130.4,130.5,130.9$, 131.5, 134.4, 135.8, 160.7, 164.7, 167.3.

\section{Vascular reactivity studies}

Male Wistar rats (250-300 g) were obtained from the Department of Physiology and Pharmacology, Federal University of Pernambuco (UFPE). They were kept under standard environmental conditions ( $12 \mathrm{~h}$ dark/light cycle) and temperature $\left(22 \pm 2{ }^{\circ} \mathrm{C}\right)$. Water and industrialized dry food (Labina ${ }^{\circledR}$, Purina, Brazil) were available ad libitum. All the experimental protocols were submitted to and approved by the UFPE's Animal Experimentation Ethics Committee, under license No. 021446/2013-12 in accordance with the National Institute of Health Guide for the Care and Use of Laboratory Animals.

The rats were killed by $\mathrm{CO}_{2}$ inhalation. The thoracic aorta was quickly removed, dissected free, and cut into $4 \mathrm{~mm}$-long rings. The aortic rings were placed between two stainless-steel stirrups and connected to an isometric force transducer, in order to measure the tension. The rings were placed in a $10 \mathrm{~mL}$ organ chamber containing Krebs solution with the following composition $\left(\mathrm{mmol} \mathrm{L}^{-1}\right): \mathrm{NaCl}$ 130, $\mathrm{KCl}$ 4.7, $\mathrm{KH}_{2} \mathrm{PO}_{4} 1.2, \mathrm{MgSO}_{4}$ 1.2, $\mathrm{NaHCO}_{3}$ 14.9, glucose 5.5, and $\mathrm{CaCl}_{2}$ 1.6. The solution was maintained at $\mathrm{pH} 7.4$, and gassed with $95 \% \mathrm{O}_{2}$ and $5 \% \mathrm{CO}_{2}$, at $37{ }^{\circ} \mathrm{C}$. The rings were initially stretched to a basal tension of $1.5 \mathrm{~g}$ and allowed to equilibrate for $60 \mathrm{~min}$ in the bath, being washed every $15 \mathrm{~min}$. The endothelium was mechanically removed and the effectiveness of the removal was assessed by the absence of relaxation induced by acetylcholine $(1 \mu \mathrm{mol} \mathrm{L}-1)$ in the presence of contractile tone induced by phenylephrine $\left(0.1 \mu \mathrm{mol} \mathrm{L}^{-1}\right)$.

Cumulative concentration-effect curves for phenylephrine $\left(0.1 \mathrm{nmol} \mathrm{L}^{-1}-10 \mu \mathrm{mol} \mathrm{L}^{-1}\right)$ were constructed in the absence or in the presence of the derivatives or Prazosin $\left(10^{2} \mu \mathrm{mol} \mathrm{L}^{-1}\right)$. When the derivatives were used, they were added to the bath $30 \mathrm{~min}$ prior to the concentration-effect curve for phenylephrine to allow the equilibrium time necessary for the molecules to bind to the target receptors.

\section{Statistical analysis}

The results of the vascular reactivity studies were expressed as mean \pm standard error of mean (S.E.M.). Differences between means were determined using one way analysis of variance (ANOVA) followed by Dunnet's multiple comparison test. The analysis was performed using GraphPad Prism 6.0 ${ }^{\circledast}$. The level of significance for rejection of the null hypothesis was set at $5 \%(p<0.05)$.

\section{Results and Discussion}

Prior to the synthesis of the 2-methylsulfanyl-6-oxo4-aryl-1,6-dihydro-pyrimidine 5-carbonitriles, it was necessary to choose and to optimize the reaction conditions. In our synthetic approach, aromatic aldehydes (1a-j) react with ethyl cyanoacetate (2) and 2-methyl-2-thiopseudourea hemisulfate (3), in the presence of potassium carbonate as base. To this end, some experiments were performed, in which we tested three parameters we believe that could generate better reaction yields or smaller reaction times: solvent, method (ultrasound or conventional stirring) and temperature. These results are depicted in Table 1. The reaction conditions in those preliminary tests were optimized with benzaldehyde.

These results clearly indicate that the best conditions employed an equal mixture of ethanol and water as solvents, using ultrasound irradiation at $50{ }^{\circ} \mathrm{C}$, showing that $4 \mathbf{a}$ can be obtained via MCR in $72 \%$, after isolation and purification. This way, we decided to perform the synthesis of a series of 2-methylsulfanyl-6-oxo-4-aryl1,6-dihydro-pyrimidine 5-carbonitriles, under the same reaction conditions (Table 2).

There are, possibly, two subsequent reactions occurring in the reaction media in order to build the heterocycle scaffold: the first one is the Knoevenagel reaction, ${ }^{29}$ where an aldehyde reacts with ethyl cyanoacetate, to form Michael's intermediate. This latter can now react with 2-methyl-2-thiopseudourea. Michael's adduct can then be attacked by the single electron pair of a nitrogen atom in the uronium portion. There is then a sequence of additions with a ring closure to form the pyrimidinone cycle. The last step in this mechanistic explanation relies on the oxidation promoted by atmospheric oxygen, resulting on the loss of a $\mathrm{H}_{2}$ molecule and leading to the heterocycle (Scheme 1).

Once those compounds are closely related to Prazosin $(\mathbf{I}),{ }^{4}$ we have decided to evaluate their vascular reactivity in isolated aorta of rats. However, those derivatives showed poor solubility in water, which could derail the tests. Therefore, we decided to synthesize the sodium salts of pyrimidinones $\mathbf{4 a - j}$. The synthesis was performed by mixing the pyrimidinones in equimolar quantity of $\mathrm{NaOH}$ dissolved in ethanol, under ultrasound irradiation (Scheme 2). Those derivatives were much more soluble in water, so we could carry out the pharmacological tests.

Phenylephrine is a selective alpha-1 adrenergic agonist. When phenylephrine binds to alpha- 1 adrenergic 
Table 1. Influence of reaction conditions on the synthesis of 2-methylsulfanyl-6-oxo-4-aryl-1,6-dihydro-pyrimidine 5-carbonitrile

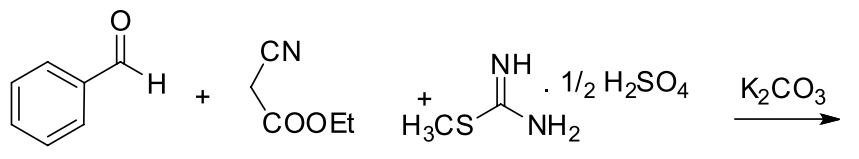

$1 \mathbf{a}$

2

3<smiles>CSc1nc(-c2ccccc2)c(C#N)c(=O)[nH]1</smiles>

\section{Solvent}

acetonitrile

water

ethanol/water

acetonitrile

water

ethanol

ethanol/water

acetonitrile

water

ethanol/water

acetonitrile

water

ethanol/water

ethanol
Yield $^{\mathrm{a}} / \%$

39

14

29

44

8

52

72

30

7

conventional

ultrasound

ultrasound

ultrasound

conventional

36

47

6

40

0

Isolated yieds.

Table 2. Ultrasound-assisted multicomponent reaction for the synthesis of 6-oxo-2,4-diaryl-1,6-dihydro-pyrimidine-5-carbonitriles (4a-j)

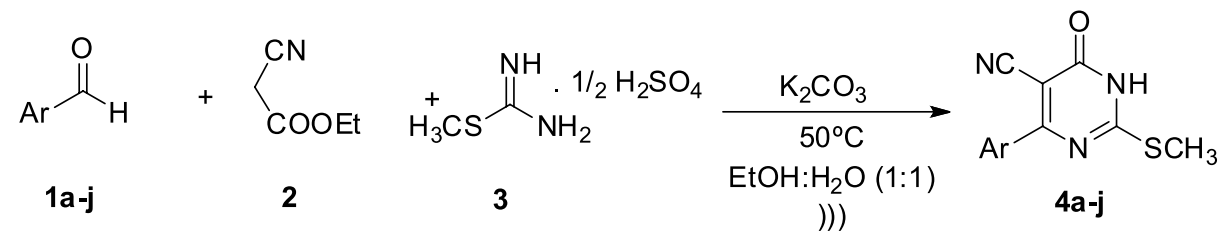

\begin{tabular}{|c|c|c|}
\hline Compound & $\mathrm{Ar}$ & Yield / \% \\
\hline $4 a$ & $\mathrm{Ph}$ & 72 \\
\hline $4 b$ & $o$-tolyl & 55 \\
\hline $4 c$ & $p$-tolyl & 50 \\
\hline $4 d$ & $p$-ClPh & 47 \\
\hline $4 e$ & $p$-BrPh & 40 \\
\hline $4 f$ & $p$-FPh & 56 \\
\hline $4 \mathrm{~g}$ & $p-\mathrm{OCH}_{3} \mathrm{Ph}$ & 55 \\
\hline $4 h$ & $m-\mathrm{NO}_{2} \mathrm{Ph}$ & 53 \\
\hline $4 i$ & $p-\mathrm{NO}_{2} \mathrm{Ph}$ & 53 \\
\hline $4 j$ & 3,4-diClPh & 40 \\
\hline
\end{tabular}

receptor, it induces a cellular cascade that leads to vascular smooth muscle cells contraction. ${ }^{1}$ It can be assessed by cumulative concentration-effect curves. Rossum and Van Den Brink ${ }^{30}$ described the construction of concentrationeffect curves. It is based on the cumulative addition of crescent concentrations of a drug to a tissue in an organ bath. From the concentration versus effect graphic, the pharmacological parameter "maximum effect" may be calculated. This parameter represents the maximum effect a drug can generate over a specific tissue.

Pharmacologic antagonists are substances that bind to the receptor and do not induce receptor activation (effect), 


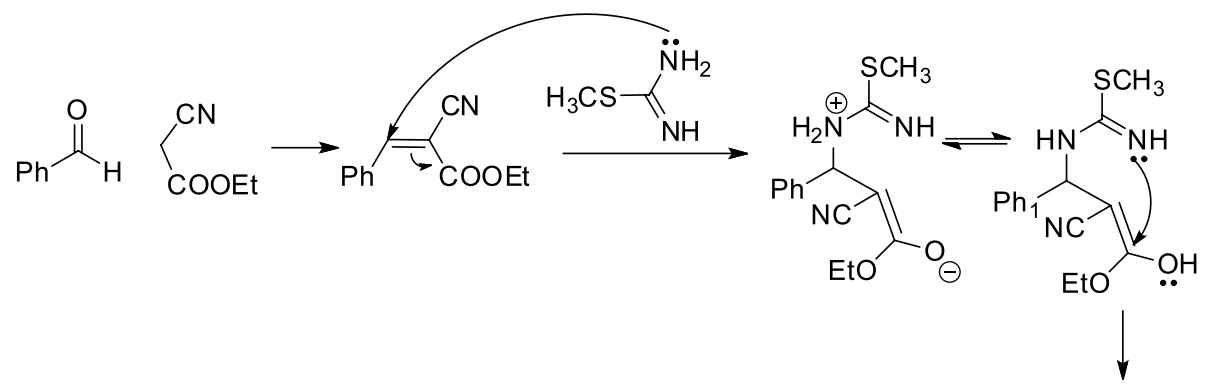

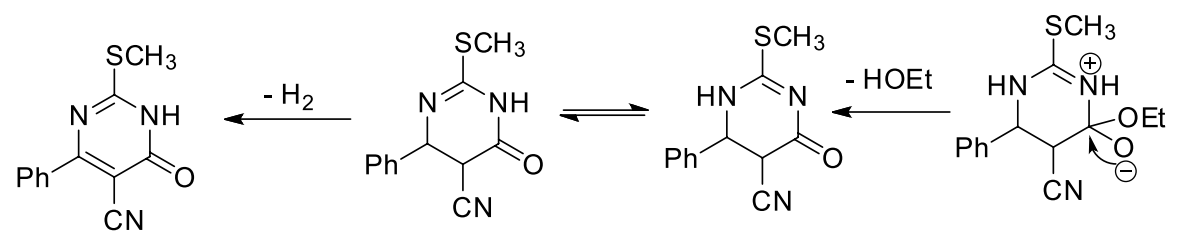

Scheme 1. Reaction mechanism for the formation of thiomethyl-pyrimidinones.

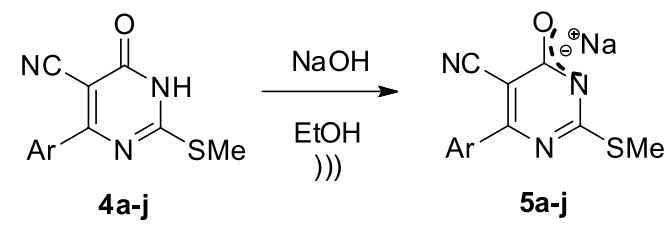

Scheme 2. Thiomethyl-pyrimidinone salts preparation.

but prevent the agonist from binding with the receptor and inducing its cellular effect. Therefore, in the presence of an antagonist, the effect of the agonist is reduced or, depending on the concentration, even absent. Prazosin, Terazosin and Doxazosin are examples of alpha-1 adrenergic antagonists. In the presence of an antagonist, the maximum effect, as well as other pharmacologic parameters in the concentration-effect curves, may be reduced. ${ }^{1}$

In this study, concentration-effect curves to phenylephrine were constructed in the absence and in the presence of the derivatives in isolated rat thoracic aorta. As it can be seen in Figure 2 and Table 3, most of the derivatives, except $\mathbf{5 b}, \mathbf{5} \mathbf{g}$, and $\mathbf{5 i}$, were able to inhibit the phenylephrine-induced contraction in thoracic aorta isolated from rats (diminish the maximum effect of the phenylephrine concentration-effect curve). It was observed that the presence of electronegative atoms in the phenyl ring has some influence in improving the biological activity. The totality of derivatives containing halogen atoms in their structures showed very good inhibition of the phenylephrine-induced contraction. These findings show that electronic effects are important for the maintenance of the pharmacological activity. In addition, steric effects seem to rule the activity once $\mathbf{5 d}(p-\mathrm{ClPh})$ and $\mathbf{5 e}(p-\mathrm{BrPh})$ showed better percentages of inhibition than $\mathbf{5 f}(p-\mathrm{FPh})$.

These results suggest that the molecules may be alpha-1 antagonists. However, more conclusive experiments should be performed to ensure this assumption.

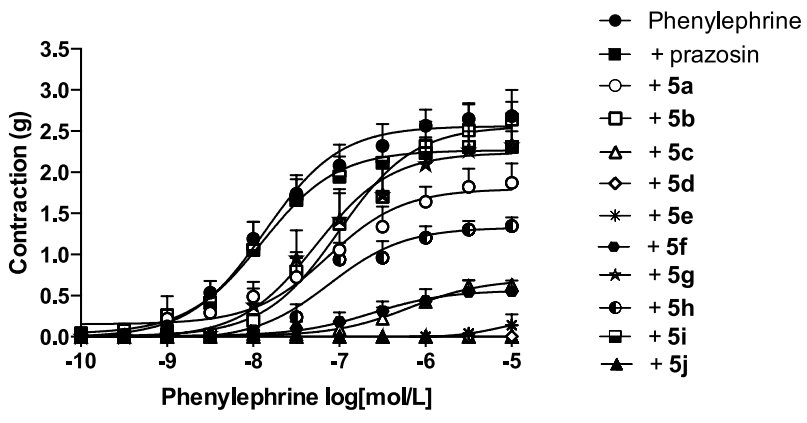

Figure 2. Contraction induced by phenylephrine in rat thoracic aorta in the absence or in the presence of pyrimidinone derivatives. Each point represents mean \pm S.E.M. of different animals. Statistically different from phenylephrine, $p<0.05$ (one way ANOVA, followed by Newmann-Keuls).

Table 3. Contraction induced by phenylephrine in rat thoracic aorta in the absence or in the presence of the derivatives

\begin{tabular}{|c|c|c|}
\hline Moiety & $\begin{array}{c}\text { Contraction of } \\
\text { phenylephrine / g }\end{array}$ & $\begin{array}{l}\text { Percentage of } \\
\text { inhibition / \% }\end{array}$ \\
\hline None & $2.68 \pm 0.17$ & - \\
\hline Prazosin $\left(100 \mu \mathrm{mol} \mathrm{L}^{-1}\right)$ & $0.00 \pm 0.00^{\mathrm{a}}$ & 100.0 \\
\hline 5a $\left(100 \mu \mathrm{mol} \mathrm{L}^{-1}\right)$ & $1.87 \pm 0.24^{\mathrm{a}}$ & 30.2 \\
\hline $\left.\mathbf{5 b}(100 \mu \mathrm{mol} \mathrm{L})^{-1}\right)$ & $2.64 \pm 0.36$ & 1.5 \\
\hline $\mathbf{5 c}\left(100 \mu \mathrm{mol} \mathrm{L}{ }^{-1}\right)$ & $0.63 \pm 0.06^{\mathrm{a}}$ & 76.5 \\
\hline 5d $\left(100 \mu \mathrm{mol} \mathrm{L}{ }^{-1}\right)$ & $0.00 \pm 0.00^{\mathrm{a}}$ & 100.0 \\
\hline $\mathbf{5 e}\left(100 \mu \mathrm{mol} \mathrm{L}{ }^{-1}\right)$ & $0.14 \pm 0.13^{\mathrm{a}}$ & 94.8 \\
\hline $\mathbf{5 f}\left(100 \mu \mathrm{mol} \mathrm{L} \mathrm{L}^{-1}\right)$ & $0.55 \pm 0.13^{\mathrm{a}}$ & 79.5 \\
\hline $\mathbf{5 g}\left(100 \mu \mathrm{mol} \mathrm{L}^{-1}\right)$ & $2.33 \pm 0.30$ & 13.0 \\
\hline $\mathbf{5 h}\left(100 \mu \mathrm{mol} \mathrm{L}{ }^{-1}\right)$ & $1.34 \pm 0.11^{\mathrm{a}}$ & 50.0 \\
\hline $\mathbf{5 i}(100 \mu \mathrm{mol} \mathrm{L}-1)$ & $2.31 \pm 0.19$ & 13.8 \\
\hline $\mathbf{5 J}\left(100 \mu \mathrm{mol} \mathrm{L} \mathrm{L}^{-1}\right)$ & $0.00 \pm 0.00^{\mathrm{a}}$ & 100.0 \\
\hline
\end{tabular}

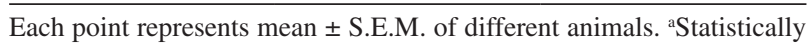
different from phenylephrine, $p<0.05$ (one way ANOVA, followed by Newmann-Keuls). 


\section{Conclusions}

In this article we advanced a multicomponent approach employing ultrasound irradiation under mild conditions to prepare a series of vasoactive pyrimidinones. This environmentally friendlier protocol furnishes products with higher global yields, employs potassium carbonate as base instead of organic harmful bases and is performed in 2 hours, which is much faster than the usual 2-day route.

Moreover, the molecules were able to inhibit the phenylephrine-induced contractions in rat thoracic aorta, suggesting that they act like alpha- 1 antagonists. However, more experiments should be performed to ensure this assumption.

\section{Supplementary Information}

Supplementary data associated with this article are available free of charge at http://jbcs.org.br as PDF file.

\section{Acknowledgments}

We thank the Brazilian agencies CNPq and FACEPE (PRONEX-0788-1.06/08) for financial support. A. N. A. is grateful to CAPES for a graduate research fellowship. $\mathrm{H}$. B. W. B. is grateful to $\mathrm{CNPq}$ for an undergraduate research fellowship.

\section{References}

1. Hoffman, B. B. In Goodman \& Gilman's The Pharmacological Basis of Therapeutics, $10^{\text {th }}$ ed.; Hardman, J. G.; Limbird, L. E., eds.; McGraw-Hill: New York, USA, 2001, ch. 10.

2. Chow, K. T.; Chan, L. W.; Heng, P. W. S.; J. Pharm. Sci. 2008, 97, 3467.

3. Piascik, M. T.; Perez, D. M.; J. Pharmacol. Exp. Ther. 1991, 298, 403.

4. Giardinà, D.; Crucianelli, M.; Gulini, U.; Marucci, G.; Melchiorre, C.; Spampinato, S.; Eur. J. Med. Chem. 1997, 32, 9.

5. Romeiro, L. A. S.; Ferreira, M. S.; Silva, L. L.; Castro, H. C.; Miranda, A. L. P.; Silva, C. L. M.; Noël, F.; Nascimento, J. B.; Araújo, C. V.; Tibiriçá, E.; Barreiro, E. J.; Fraga, C. A. M.; Eur. J. Med. Chem. 2011, 46, 3000.

6. Hancock, A. A.; Brune, M. E.; Witte, D. G.; Marsh, K. C.; Katwala, S.; Milicic, I.; Ireland, L. M.; Crowell, D.; Meyer, M. D.; Kerwin Jr, J. F.; J. Pharmacol. Exp. Ther. 1998, 285, 628.

7. Stringfellow, D. A.; Adv. Enzyme Regul. 1981, 19, 335.

8. Scheringa, M.; Ijzermans, J. N.; Jeekel, J.; Marquet, R. L.; Cancer Immunol. Immunother. 1990, 32, 251.

9. Vroegop, S. M.; Chapman, D. L.; Decker, D. E.; Galinet, L. A.;
Brideau, R. J.; Ready, K. A.; Dunn, C. J.; Buxser, S. E.; Int. J. Immunopharmacol. 1999, 21, 647.

10. Saladino, R.; Ciambecchini, U.; Maga, G.; Mastromarino, P.; Conti, C.; Botta, M. A.; Bioorg. Med. Chem. Lett. 2002, 10, 2143.

11. Salimbeni, A.; Canevotti, R.; Paleari, F.; Poma, D.; Caliari, S.; Fici, F.; Cirillo, R.; Renzetti, A. R.; Subissi, A.; Belvisi, L.; Bravi, G.; Scolastico, C.; Giachetti, A.; J. Med. Chem. 1995, 38, 4806.

12. Yamaguchi, M.; Wakasugi, K.; Saito, R.; Adachi, Y.; Yoshikawa, Y.; Sakurai, H.; Katoh, A.; J. Inorg. Biochem. 2006, 100, 260.

13. White, D. C.; Greenwood, T. D.; Downey, A. L.; Bloomquist, J. R.; Wolfe, J. F.; Bioorg. Med. Chem. 2004, 12, 5711.

14. Bruno, O.; Ranise, A.; Bondavalli, F.; Schenone, S.; D’Amico, M.; Falciani, M.; Filippelli, W.; Rossi, F.; Farmaco 1994, 49, 551.

15. dos Anjos, J. V.; Srivastava, R. M.; Costa-Silva, J. H.; Scotti, L.; Scotti, M. T.; Wanderley, A. G.; Leite, E. S.; Melo, S. J.; Mendonça Junior, F. J. B.; Molecules 2012, 16, 809.

16. dos Anjos, J. V.; Mendonça Jr, F. J. B.; Costa-Silva, J. H.; de Souza, I. A.; Melo, S. J.; Lat. Am. J. Pharm. 2008, 27, 343.

17. Brown, D. J. In Comprehensive Heterocyclic Chemistry, vol. 3; Katritzky, A. R.; Rees, C. W., eds.; Pergamon Press: Oxford, United Kingdom, 1984.

18. Kappe, C. O.; Eur. J. Med. Chem. 2000, 35, 1043.

19. Melo, S. J.; Luu-Duc, C.; Thomasson, F.; Narcisse, G.; Gaultier, C.; Ann. Pharm. Fr. 1992, 50, 39.

20. Wierenga, W.; Pharmacol. Ther. 1985, 30, 67.

21. Sheibani, H.; Seifi, M.; Bazgir, A.; Synth. Commun. 2009, 39, 1055.

22. Dömling, A.; Ugi, I.; Angew. Chem. Int. Ed. 2000, 39, 3168.

23. Deligeorgiev, T.; Kaloyanova, S.; Lesev, N.; Vaquero, J. J.; Ultrason. Sonochem. 2010, 17, 783.

24. Memarian, H. R.; Farhadi, A.; Ultrason. Sonochem. 2008, 15, 1015.

25. Patil, R.; Bhoir, P.; Deshpande, P.; Wattamwar, T.; Shirude, M.; Chaskar, P.; Ultrason. Sonochem. 2013, 20, 1327.

26. Enrique, R.; Hortensia, R.; Julieta, C.; Esperanza, S.; Margarita, S.; Roberto, M.-A.; Nazario, M.; Ultrason. Sonochem. 2011, $18,32$.

27. Buriol, L.; München, T. S.; Frizzo, C. P.; Marzari, M. R. B.; Zanatta, N.; Bonacorso, H. C.; Martins, M. A. P.; Ultrason. Sonochem. 2013, 20, 1139.

28. Pagadala, R.; Maddila, S.; Jonnalagadda, S. B.; Ultrason. Sonochem. 2014, 21, 472.

29. Xavier, A. L.; Simas, A. M.; Falcão, E. P. S.; dos Anjos, J. V.; Tetrahedron Lett. 2013, 54, 3462.

30. Rossum, V.; Van Den Brink, F. G.; Arch. Int. Pharmacodyn. Ther. 1963, 143, 240.

Submitted: August 11, 2016 Published online: November 3, 2016 